\title{
Exciton-enhanced recombination in silicon at high concentrations of charge carriers
}

\author{
A.V. Sachenko, A.P. Gorban, V.P. Kostylyov \\ Institute of Semiconductor Physics of NASU, 45, prospect Nauki, 03028, Kyiv, Ukraine
}

\begin{abstract}
The interrelation between processes of electron-hole recombination and annihilation of excitons in silicon is examined. It is shown, that recombination processes can be essentially influenced by the exciton annihilation at high concentrations of non-equilibrium or equilibrium charge carriers. In $n$-type material a correlation between Shockley-Reed-Hall lifetime values and a square-law recombination coefficient is found. This correlation is explained in terms of assumption that both Shockley-Reed-Hall lifetime, and non-radiative exciton annihilation time constant responsible for a square-law recombination, are determined by the same deep level. It is stated, that the mentioned regularities should essentially affect the bulk lifetime values in $n$-type silicon at doping concentration exceeding $10^{16} \mathrm{~cm}^{-3}$.
\end{abstract}

Keywords: exciton-enhanced recombination, excitons in silicon.

Paper received 17.12.99; revised manuscript received 04.01.00; accepted for publication 06.01.00.

\section{Introduction}

Due to technology improvements and usage of perfect mono-crystalline silicon with high lifetime of minority charge carriers the conversion efficiency of silicon solar cells (SC), $\eta$, was enhanced up to $24 \%$ in AM1.5 standard terrestrial conditions [1]. It was shown, that in such high-efficiency SC $\eta$ value can be affected not only by Shockley-Reed-Hall recombination but also by both band-to-band photon-emitting and Auger recombination mechanisms. In a number of papers [2-9] was shown, that generation-recombination processes in silicon can be influenced by effects, particularly, exciton-related, resulting from multi-particle interaction of charge carriers. In the present paper is shown, that non-equilibrium phenomena analyzed in [2-9], do not exhaust all possible mechanisms for exciton participation in recombination of nonequilibrium charge carriers in silicon. In particular, due to examination of experimental results $[4,5,10-16]$ related to investigation of bulk lifetime change with concentration of mobile charge carriers, a correlation between Shockley-Reed-Hall lifetime, $\tau_{r}$, and square-law recombination coefficient, $A$, was found in $n$-type silicon. The results obtained testify to essential role of non-radiating exciton annihilation performing as Auger recombination with participation of deep impurity centers.

\section{Theory}

According to approach developed in [17-19], a continuity equations for excess free electron-hole and exciton fluxes generated in the semiconductor by monochromatic radiation, in neglect effects of highest orders [20], can be written as:

$$
\begin{aligned}
& D_{A} \frac{d^{2} \Delta n}{d x^{2}}-\left[\frac{1}{\tau_{r n}}+A_{0}\left(n_{0}+\Delta n\right)+\right. \\
& \left.+C_{n}\left(n_{0}+\Delta n\right)^{2}+C_{p}\left(n_{0}+\Delta n\right) \Delta n\right] \times \Delta n- \\
& -\gamma_{1}\left(n_{0}+\Delta n\right) \Delta n+\gamma_{2} n_{x}+\alpha I_{0} \exp (-\alpha x)=0, \\
& D_{x} \frac{d^{2} \Delta n_{x}}{d x^{2}}-\frac{\Delta n_{x}}{\tau_{x}}+\gamma_{1}\left(n_{0}+\Delta n\right) \Delta n-\gamma_{2} n_{x}=0 .
\end{aligned}
$$

Here $\Delta n$ is a concentration of excess electron-hole pairs in the sample bulk, $\tau_{r n}$-Shockley-Reed-Hall lifetime, $A_{0}$ - a square-law recombination coefficient, related both to photon-emitting band-to-band recombination and to impurity-assisted Auger recombination [21], $C_{n}$ and $C_{p}$ - parameters of the band-to-band Auger recombination for electrons and for holes, $\gamma_{1}-$ a probability for electron-hole pair coupling into excitons, $\gamma_{2}-\mathrm{a}$ probability for exciton dissociation, $\tau_{x}$ - lifetime determined by annihilation of excitons, $n_{x}$ and $\Delta n_{x}$ - total 


\section{A.V. Sachenko et al.: Exciton-enhanced recombination in silicon at high ...}

and excess concentrations of excitons in a sample, $I_{0}$-intensity of light penetrated into a sample, $\alpha$ - light absorption coefficient, $D_{A}$ and $D_{x}$ - coefficients of an ambipolar diffusion of electron-hole pairs and diffusion of excitons, $n_{0}$ - equilibrium concentration of electrons in the bulk of ntype silicon. At a spelling of equations (1) and (2) it was supposed, that $\Delta n_{x} \cong n_{x}$, and the non-equilibrium hole concentration $p$ considerably exceeds an equilibrium value $p_{0}$, and is equal to an excess concentration of electrons $\Delta n$. Besides, it was assumed that optical generation occurs due to transitions in the region of band-to-band absorption.

The total time of exciton annihilation, $\tau_{x}$, can be defined by addition of inverse times related to photo-emitting, $\tau_{x}^{r}$, and non-radiating, $\tau_{x}^{n}$, annihilation of excitons, i.e.: $\frac{1}{\tau_{x}}=\frac{1}{\tau_{x}^{r}}+\frac{1}{\tau_{x}^{n}}$.

The set of equations (1) - (3) should be supplemented by appropriate boundary conditions:

$$
\begin{aligned}
& j_{n}(x=0, d)=-\left.D_{A} \frac{d \Delta n}{d x}\right|_{x=0, d}= \\
& =\mp S_{0, d} \cdot \Delta n(x=0, d) \\
& j_{x}(x=0, d)=-\left.D_{x} \frac{d \Delta n_{x}}{d x}\right|_{x=0, d}= \\
& =\mp S_{0, d}^{x} \cdot \Delta n_{x}(x=0, d)
\end{aligned}
$$

where $S_{0, d}$ and $S_{0, d}^{x}$-effective velocities of electron-hole recombination and exciton annihilation at surfaces located at $x=0, d$. Note, that similar to (1) and (2) set of equations was solved in [8] for the case of linear excitation level with taking into account the generation in exciton absorption region.

The time of exciton dissociation in silicon, $\gamma_{2}^{-1}$, is approximately equal to $10^{-12} \mathrm{~s}$ at $T=300 \mathrm{~K}$ [9], while the time of their annihilation essentially exceeds the mentioned value $[22,23]$. For this reason the solution of the equation (2) can be written as:

$$
n_{x} \cong \frac{\gamma_{1}}{\gamma_{2}}\left(n_{0}+\Delta n\right) \Delta n
$$

On the other hand, according to the approach, developed in [7-9],

$$
n_{x} \cong \frac{1}{n^{\bullet}}\left(n_{0}+\Delta n\right) \Delta n,
$$

where

$$
n^{\bullet}=\left(\frac{N_{c} N_{v}}{N_{x}}\right) \exp \left(-\frac{E_{x}}{k T}\right),
$$

$N_{c}, N_{v}$ and $N_{x}$-effective densities of states for electrons, holes and excitons, $E_{x}$ - exciton binding energy. Comparing (6) and (7), we obtain:

$$
\frac{\gamma_{2}}{\gamma_{1}}=n^{\bullet}
$$

The quantity $n^{\bullet}$ for silicon at a room temperature was evaluated in [7] and was shown to be $n^{\bullet} \approx 8 \cdot 10^{17} \mathrm{~cm}^{-3}$ in approximation of negligible screening of a Coulomb interaction between electrons and holes. With taking the screening into consideration, the energy of a basic state of Motttype exciton in a non-degenerate semiconductor can be represented similarly to [8] as:

$E_{x}{ }^{*}=E_{x}\left[1-\left(\frac{n_{0}+2 \Delta n}{n_{M}}\right)^{1 / 2}\right]^{2}$,

where $n_{M}$ - critical concentration, at which Mott transition for excitons occurs $\left(n_{M} \approx 10^{18} \mathrm{~cm}^{-3}\right.$ in silicon at $T=$ $300 \mathrm{~K})$. At room temperatures given by (10) regularities do not result in strong change of $n$ value. However, the change can be essential at low temperatures.

Combining the equations (1) and (2) with taking into account (6) and (9) gives:

$$
\begin{aligned}
& D_{A} \frac{d^{2} \Delta n}{d x^{2}}-\left[\frac{1}{\tau_{r n}}+A_{0}\left(n_{0}+\Delta n\right)+\right. \\
& \left.+C_{n}\left(n_{0}+\Delta n\right)^{2}+C_{p}\left(n_{0}+\Delta n\right) \Delta n\right] \times \Delta n+ \\
& +\frac{D_{x}}{n^{\bullet}}\left[\left(n_{0}+2 \Delta n\right) \frac{d^{2} \Delta n}{d x^{2}}+2\left(\frac{d \Delta n}{d x}\right)^{2}\right]- \\
& -\frac{1}{n^{\bullet} \tau_{x}}\left(n_{0}+\Delta n\right) \Delta n+\alpha I_{0} \exp (-\alpha x)=0 .
\end{aligned}
$$

The estimations carried out with usage of values $D x=$ $17 \mathrm{~cm}^{2} / \mathrm{s}$ [7] and $D_{A}=18 \mathrm{~cm}^{2} / \mathrm{s}$ show, that at $\Delta n>10^{17}$ $\mathrm{cm}^{-3}$ the concentration of excitons becomes comparable to concentration of electron-hole pairs. Essential also there is a contribution of exciton component to a total diffusion flux, while the equation (11) becomes non-linear.

In [23] was noted, that as the quantum yield of exciton luminescence in silicon is about $0.01 \%$, it means, that the main mechanism of exciton annihilation is the nonradiating channel. If we assume $\tau_{x}=10^{-7} \mathrm{~s}$ [22], than the value of $1 / n^{\bullet} \tau_{x} \approx 10^{-11} \mathrm{~cm}^{3} \mathrm{~s}^{-1}$ can be obtained. This quantity is four order of magnitude more high, than a value of band-to-band photon-emitting recombination constant [24]. Therefore annihilation of excitons can rather essentially affect an effective lifetime of electron-hole pairs, and its dependence on concentration appears to follow square-law relation. For an explanation of low values of a non-radiating exciton annihilation time the mechanism of Auger recombination was attracted [5, 6, 23, 25, 26].

For further estimations we shall use a simple approach offered in [5]. This approach is based on the assumption, that under exciton formation a high local density of electron-hole pairs $n_{L} \approx 1 /(4 / 3) \pi a_{B}^{3}$ appears, where $a_{B}$ is the Bohr radius of an exciton. So, having substituted $a_{B}$ value for silicon, equal to $4.2 \mathrm{~nm}$, we obtain $n_{L}=3.25 \cdot 10^{18} \mathrm{~cm}^{-3}$. As this concentration is high enough for exhibiting of Auger recombination, in particular, with participation of deep im- 


\section{A.V. Sachenko et al.: Exciton-enhanced recombination in silicon at high ...}

purity centers, the mentioned recombination can be responsible for non-radiating annihilation of excitons. In this case the following relation is valid:

$$
1 / \tau_{x}^{n}=n_{L} G N_{t}
$$

where $G$ is a constant of the impurity-assisted Auger recombination, and $N_{t}$ is a concentration of deep impurity centers.

In the case of homogeneous excitation, when the surface recombination is small enough and does not affect an effective lifetime, it is possible to write:

$\Delta n=\alpha I_{0} \cdot \tau_{e f}$,

where

$$
\begin{aligned}
& \frac{1}{\tau_{e f}}=\left[\frac{1}{\tau_{r n}}+A\left(n_{0}+\Delta n\right)+\right. \\
& \left.+C_{n}\left(n_{0}+\Delta n\right)^{2}+C_{p}\left(n_{0}+\Delta n\right) \Delta n\right] \\
& A=A_{0}+\frac{1}{n}\left(\frac{1}{\tau_{x}^{r}}+n_{L} G N_{t}\right)
\end{aligned}
$$

\section{Analysis of experimental data and discussion}

We shall analyze now experimental results concerning dependence of effective recombination lifetime, $\tau_{e f}$, on bulk concentration of mobile charge carriers in silicon $[4,5,10-16]$. In the majority of cited publications $\tau_{e f}$ was determined from transient response of IR flux modulated by photon absorption on mobile charge carriers inside semiconductor. Initial concentration of mobile carriers in the sample was changed either by optical excitation, or by variation of doping impurity concentration. At processing experimental results the formula (14) was utilized in the case of $n$-type silicon, and formula

$$
\begin{aligned}
& \frac{1}{\tau_{e f}}=\left[\frac{1}{\tau_{r p}}+A\left(n_{0}+\Delta n\right)+\right. \\
& \left.+C_{p}\left(n_{0}+\Delta n\right)^{2}+C_{n}\left(n_{0}+\Delta n\right) \Delta n\right]
\end{aligned}
$$

in the case of p-type silicon, where $\tau_{r p}$ is Shockley-ReedHall lifetime in a $p$-type semiconductor. At comparison of experimental data and theoretical curves the band-toband Auger recombination coefficients, $C_{n}$ and $C_{p}$, were utilized as fitting parameters, while $\tau_{r}$ values were taken directly from appropriate experimens. Besides, to specify the shape of concentration dependence of Auger recombination coefficient for electrons, $C_{n}$, experimental curve $C_{n}(n)$ [3] was analyzed, Fig. 1 . It was established, that in n-type silicon a good agreement of experimental and calculated $C_{n}(n)$ curves can be obtained at usage of the empirical formula

$$
C_{n}=\left(2.8 \cdot 10^{-31}+\frac{2.5 \cdot 10^{-22}}{\left(n_{0}+\Delta n\right)^{1 / 2}}\right) \mathrm{cm}^{6} / \mathrm{s} \text {. }
$$

Note, that $C_{n}(n)$ relation shown in [3] for $T=70 \mathrm{~K}$, can be circumscribed by the following expression:

Table

\begin{tabular}{llll}
\hline \hline$\tau, \mathrm{s}$ & $A, \mathrm{~cm}^{3} / \mathrm{s}$ & $\tau_{r} \cdot \mathrm{A}, \mathrm{cm}^{3}$ & Comments \\
\hline $1.8 \cdot 10^{-5}$ & $1.5 \cdot 10^{-11}$ & $2.7 \cdot 10^{-16}$ & $n$-type [10] \\
\hline $3.8 \cdot 10^{-2}$ & $2.5 \cdot 10^{-15}$ & $0.95 \cdot 10^{-16}$ & $n$-type [11], orient.<111> \\
\hline $5 \cdot 10^{-3}$ & $3 \cdot 10^{-14}$ & $1,5 \cdot 10^{-16}$ & $n$-type [11], orient.<100> \\
\hline $9 \cdot 10^{-3}$ & $3 \cdot 10^{-14}$ & $2.7 \cdot 10^{-16}$ & $n$-type [13] \\
\hline $3.47 \cdot 10^{-3}$ & $1.3 \cdot 10^{-13}$ & $4.5 \cdot 10^{-16}$ & $n$-type [14] \\
\hline $9 \cdot 10^{-3}$ & $3 \cdot 10^{-14}$ & $2.7 \cdot 10^{-16}$ & $n$-type [4], $T=80 \mathrm{~K}$ \\
\hline $2 \cdot 10^{-3}$ & $1.1 \cdot 10^{-12}$ & $p$-type [5] \\
\hline $2 \cdot 10^{-6}$ & $5 \cdot 10^{-14}$ & $2.2 \cdot 10^{-15}$ & $p$-type [15] \\
\hline $1.1 \cdot 10^{-7}$ & $1.1 \cdot 10^{-12}$ & $10^{-19}$ & $p$-type [4] \\
\hline $1.3 \cdot 10^{-3}$ & $10^{-13}$ & $1.21 \cdot 10^{-19}$ & $p$-type [4], $T=80 \mathrm{~K}$ \\
\hline $1.1 \cdot 10^{-4}$ & $1.2 \cdot 10^{-12}$ & $1.3 \cdot 10^{-16}$ & $p$-type [13] \\
\hline $6.2 \cdot 10^{-4}$ & $3 \cdot 10^{-15}$ & $1.3 \cdot 10^{-16}$ & $p$-type [15] \\
\hline $7 \cdot 10^{-3}$ & $3 \cdot 10^{-15}$ & $1.86 \cdot 10^{-18}$ & $p$-type [12] \\
\hline $5 \cdot 10^{-3}$ & $2.5 \cdot 10^{-15}$ & $2.1 \cdot 10^{-17}$ & $p$-type [12] \\
\hline $2 \cdot 10^{-3}$ & $10^{-14}$ & $1.25 \cdot 10^{-17}$ & $p$-type [12] \\
\hline $2 \cdot 10^{-4}$ & $4 \cdot 10^{-14}$ & $2 \cdot 10^{-17}$ & $8 \cdot 10^{-18}$ \\
\hline \hline
\end{tabular}




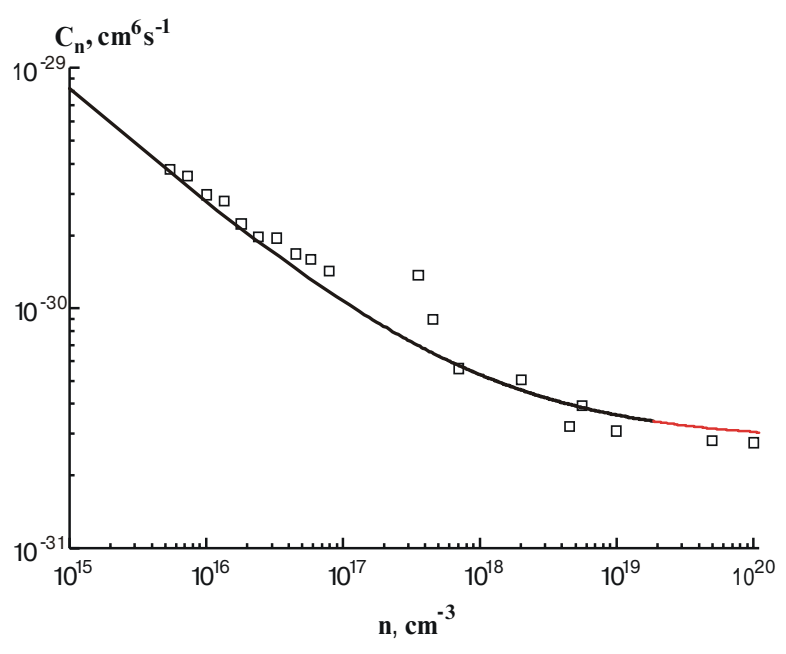

Fig.1. Concentration dependence of the Auger recombination coefficient for electrons $C_{n}$ (dotes, [3]), and its approximation by the formula (17) (line).

$$
C_{n}=\left(2.3 \cdot 10^{-31}+\frac{1.3 \cdot 10^{-12}}{n_{0}+\Delta n}\right) \mathrm{cm}^{6} / \mathrm{s} .
$$

Experimental and calculated for various concentrations of charge carriers (either equilibrium, or non-equilibrium) $\tau_{e f}^{-1}$ values are plotted in Fig. 2 . It is seen, that in $n$-type silicon a good agreement of experimental and calculated $\tau_{e f}^{-1}$ values exists in a wide range of concentration change, if given by (17) $C_{n}(n)$ relation is used. On the contrary, in a $p$-type silicon the agreement is observed only at fixed value of parameter $C_{p} \approx(1 \div 1.6) \cdot 10^{-31} \mathrm{~cm}^{6} / \mathrm{s}$.

Experimental values of Shockley-Reed-Hall lifetime $\tau_{r}$, as obtained in a variety of papers for $n$ - and $p$-type silicon samples (in particular, at $T=80 \mathrm{~K}$ [4]), are given in the Table. Here also given are the values of both square- law recombination coefficient $A$ and $\tau_{r} A$ product, at which theoretical and experimental curves might be fitted in the best way. It is seen, that the lowest experimental $A$ values are about $\approx 3 \cdot 10^{-15} \mathrm{~cm}^{-3} / \mathrm{s}$. Close to indicated $A$ value was obtained theoretically in [2], where only the photon-emitting exciton annihilation was taken into account. Thus, as obtained in [24] coefficient for photon-emitting bandto-band recombination is equal to $1.5 \cdot 10^{-15} \mathrm{~cm}^{3} / \mathrm{s}$ (in neglecting exciton effects), by substituting $A \approx 3 \cdot 10^{-15} \mathrm{~cm}^{-3} / \mathrm{s}$ into (15) it is possible to estimate the time of photon-emitting exciton annihilation at a room temperature: $\tau_{x}^{r} \approx 10^{-3} \mathrm{~s}$. In $n$-type samples $A$ values essentially exceed (except for one case) the quantity $3 \cdot 10^{-15} \mathrm{~cm}^{-3} / \mathrm{s}$. It means that in the majority cases a non-radiating annihilation of excitons dominates in $n$-type silicon. Moreover, as the $\tau_{r} A$ product in $n$-type silicon at $T=300 \mathrm{~K}$ is nearly constant and equal to $(3 \pm 1.5) \cdot 10^{-16} \mathrm{~cm}^{-3}$ (see also Fig. 3 ), the same deep centers can be responsible both for Shockley-ReedHall recombination and non-radiating exciton annihilation, on one hand, and for bulk generation within close to surface field-induced non-equilibrium depletion layer [27], on the other hand. These centers are energetically located closely to the middle of forbidden gap and have approximately equal capture coefficients for electrons and holes $\left(\approx 10^{-9} \mathrm{~cm}^{3} / \mathrm{s}\right)$. Note, that accordingly to [28], $G$ value weekly depends on the nature of deep centers, and is affected, mainly, by their energetic position.

For illustration, we shall analyze experimental data obtained in [5] for the case, when one deep Au-related acceptor level with energy $E_{a}=E_{c}-0.55 \mathrm{eV}$ and capture coefficient for holes $2 \cdot 10^{-9} \mathrm{~cm}^{3} / \mathrm{s}$ is responsible for recombination. It is seen from Fig. 3 of [5], that in the case of low excitation level a lifetime value of $3 \cdot 10^{-7} \mathrm{~s}$ at electron concentration $1.1 \cdot 10^{17} \mathrm{~cm}^{-3}$ is valid, and the effective capture coefficient $C^{\bullet}$, related to exciton-enhanced Auger recombination, is equal to $5 \cdot 10^{-8} \mathrm{~cm}^{3} / \mathrm{s}$. By use of

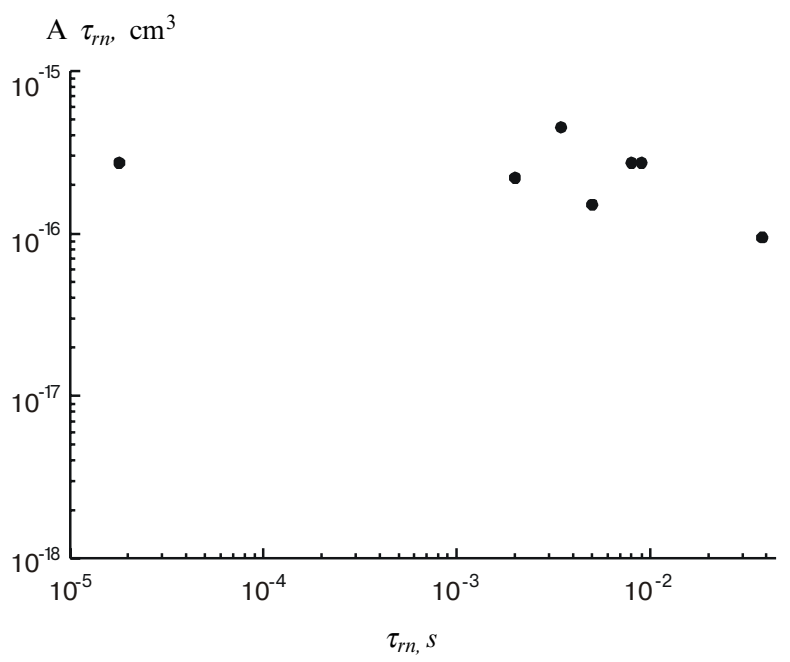

Fig. 3. Change of the product of a square-law recombination coefficient $A$ on Shockley-Reed-Hall lifetime $\tau_{r n}$ with variation of $\tau_{r n}$ value at $T=300 \mathrm{~K}$.
Fig. 2. Theoretical (lines) and experimental (points) concentration dependencies of an inverse value of bulk minority carrier lifetime ( $\bullet$ and $\bigcirc$-[11], $\mathbf{\square}$ and $\square-[4], \boldsymbol{\Delta}-[10]$ ). 


\section{A.V. Sachenko et al.: Exciton-enhanced recombination in silicon at high ...}

formulae $C^{*}=\frac{1}{\tau_{r} N_{t}}$ and $\frac{1}{\tau_{r}}=\frac{n_{L} G N_{t} n_{0}}{n^{\bullet}}$, it is possible to determine quantities $G$ and $N_{t}$, which appear to be $9.7 \cdot 10^{-26} \mathrm{~cm}^{6} / \mathrm{s}$ and $6.7 \cdot 10^{13} \mathrm{~cm}^{-3}$, respectively. So, in last case a low-signal lifetime is also determined by excitonenhanced Auger recombination, that was convincingly verified in papers $[5,6]$.

Reduced in the table low-signal lifetime values correspond to a case when the concentrations of both doping impurities and recombination centers are low, i.e. when the usual Shockley-Reed-Hall recombination mechanism is dominant. For example, having taken $\tau_{r}=9 \cdot 10^{-3} \mathrm{~s}[4]$

from the table, by use of the formula $\frac{1}{\tau_{r}}=C_{p t} N_{t_{1}}$ one can find, that at $C_{p t}=2 \cdot 10^{-9} \mathrm{~cm}^{3} / \mathrm{s}$ and $N_{t 1}=5.6 \cdot 10^{10} \mathrm{~cm}^{-3}$. The quantity $A$, obtained with taking into account the defined above $G$ and $N_{t 1}$ values, appears to be equal to $2.5 \cdot 10^{-14} \mathrm{~cm}^{3} / \mathrm{s}$. That is close to the value $3 \cdot 10^{-14} \mathrm{~cm}^{3} / \mathrm{s}$ determined experimentally on the base of [4]. The product $\tau_{r} A$ thus is equal $2.25 \cdot 10^{-16} \mathrm{~cm}^{3}$, that is also in agreement with experiment [4].

If $C_{p}$ and $G$ are not dependent on temperature, than the temperature change of $A$ is wholly defined by $n^{\bullet}(T)$

dependence and can be written as $B \exp \left(\frac{E_{x}}{k T}\right) / T^{3 / 2}$, where $B$ is temperature-independent constant. The calculated ratio of $A$ values at $80 \mathrm{~K}$ and $300 \mathrm{~K}$, thus, is equal to 35 . The experimental ratio in this case, as seen from the table, is equal to 36 , that practically coincides with the calculated one. At last, plotted in Fig. 3 of [5] experimental data, related to Au-doped $n$-type silicon, also allow to find the indicated ratio. It appears to be 33, that is also in good agreement with calculated result. Thus, practically all available for $n$-type silicon experimental data can be explained in terms of the discussed above non-radiating exciton annihilation mechanism. Note, that as follows from the comparison of the second item in (18) and shown in the table $A$ value (related to $n$-type silicon at $80 \mathrm{~K}[4]$ and equal to $1.1 \cdot 10^{-12} \mathrm{~cm}^{3} / \mathrm{s}$ ), the most probable in this case appears to be exciton-enhanced band-to-band Auger recombination mechanism.

Another regularities are observed in a p-type silicon. At high enough $\tau_{r}$ values in this material photon-emitting exciton annihilation appears to be dominant. That is evident from the value of a constant $A$, obtained in paper [2] with taking into consideration exciton effects. But in that cases, when the non-radiating exciton annihilation becomes dominant, the value of the $\tau_{r} A$ product, as a rule, appears to be much less, than in $n$-type silicon. As follows from [28], it is due to the fact, that in $p$-type silicon the probability of impurity-assisted Auger recombination is much lower, than in $n$-type. The especially low $\tau_{r} A$ product values are observed is a highly-doped $p$-type silicon [5] at $p_{0}>10^{18} \mathrm{~cm}^{-3}$, when Mott transition becomes responsible for exciton disappearance. It should be noted, that responsible for a square-law recombina- tion exciton-related effects were observed also in other semiconductors [19, 23, 25, 26].

\section{Conclusions}

Thus, as shown in the present work, the non-radiating exciton annihilation with participation of impurity-assisted Auger recombination can be responsible for a square-law recombination in $n$-type silicon in the majority of cases. Due to that the performance of silicon-based photoelectric devices operating in a high-injection mode can be essentially influenced by non-radiating exciton annihilation not only at high, but also at low $\tau_{r}$ values. Really, in view of the obtained correlation an effective lifetime in neglect by the Auger recombination can be represented as $\tau_{r} /\left(1+\vartheta_{n} \cdot 10^{-16}\left(n_{0}+\Delta n\right)\right)$, where $\vartheta_{n} \approx 3 \pm 1.5$. Thus, at $n_{0}>10^{16} \mathrm{~cm}^{-3}$ bulk lifetime value becomes much less than Shockley-Reed-Hall lifetime, and this circumstance should be taken into account. On the other hand, similar regularities should be observed not only in silicon, but also in other wide-gap semiconductors in which excitons exist at room temperatures.

\section{References}

1. A.Wang, J.Zhao and M.A.Green. $24 \%$ efficient silicon solar cells // Appl. Phys. Lett., 57 (6), pp.602-604 (1990)

2. M.Ruff, M.Fick, R.Lindner, U.Rossler, R.Heilig. The spectral distribution of the intrinsic radiative recombination in silicon // J. Appl. Phys., 74 (1), pp.267-274 (1993)

3. A.Hangleiter and R.Hacker. Enhancement of band-to-band Auger recombination by electron-hole correlations // Phys. Rev. Lett., 65 (2), pp.215-218 (1990)

4. R.Hacker and A.Hangleiter. Intrinsic upper limits of the carrier lifetime in silicon // J. Appl. Phys., 75 (11), pp.7570-7572 (1994)

5. A.Hangleiter. Non-radiative recombination via deep impurity levels in silicon: Experiment // Phys. Rev. B., 35 (17), pp.9149-9160 (1987)

6. A.Hangleiter. Non-radiative recombination via deep impurity levels in semiconductors: The exiton Auger mechanism // Phys. Rev. B., 37 (5), pp.2594 -2604 (1988)

7. D.E.Kane, R.M.Swanson. The effect of excitons on apparent band gap narrowing and transport in semiconductors // J. Appl. Phys., 73 (3), pp.1193-1197 (1993)

8. R Corkish, Daniel S-P Chan and M.A.Green. Excitons in silicon diodes and solar cells: A three-particle theory // $\mathrm{J}$. Appl. Phys., 79 (1), pp.195-203 (1996)

9. M.A.Green. Excitons in silicon solar cells: room temperature distributions and flows. In: The 2nd World Conference and Exhibition on Photovoltaic Solar Energy Conversion, 610 July 1998, Vienna, Austria, pp.74-76

10. V.A.Zuev, V.G.Litovchenko, G.A.Sukach. Many-particle recombination processes at silicon and germanium surfaces // Sov. FTP, 9 (9), pp.1641-1648 (1975)

11. E.Yablonovitch and T.Gmitter. Auger recombination in silicon at low carrier densities // Appl. Phys. Lett., 49 (10), pp. 587-590 (1986)

12. S.K.Pang and A.Rohatgi. Record high recombination lifetime in oxidized magnetic Czochralski silicon // Appl. Phys. Lett., 59 (2), p.195 (1991)

13. J.Linnross. Carrier lifetime measurements using free carrier absorption transient. Principle and injection dependence // J.Appl.Phys., 84(1), pp.275-283 (1998) 
14. G.Augustine, A.Rohatgi, N.M.Jokerst. Base doping optimization for radiation-hard $\mathrm{Si}, \mathrm{GaAs}$, and InP solar cells // IEEE Trans. Electron. Dev., ED-39 (10), pp.2395-2400 (1992)

15. R.R.King, R.A.Sinton, R.M.Swanson. Front and back surface fields for point-contact solar cells. In: The $20^{\text {th }}$ IEEE Photovoltaic Spec. Conf., Las Vegas, Nev., Sept.26-30,1988. Conf. Rec. Vol.1.-New York, N.Y.-1988. pp.538-544

16. S.K.Pang and A.Rohatgi. A new methodology for separation Shockley-Read-Hall lifetime and Auger recombination coefficients from the photo-conductivity decay technique // J.Appl. Phys., 74 (9), pp.5554-5560 (1993)

17. A.V.Sachenko, V.A.Tyagai and A.G.Kundzich. Exciton luminescence in semiconductors. Surface recombination and space-charge-layer effects // Phys. Stat. Sol., B88, pp.797804 (1978)

18. M.A.Green. Silicon solar cells: Advanced principles and practice. Ed. by Bridge Printery, Sydney, 1995

19. E.L.Nolle. On the recombination via exciton states in semiconductors // Sov. FTT, 9, p.90-96 (1967)

20. C.B. a la Guillaume, J.-M. Debever, and F.Salvan. Radiative recombination in highly excited CdS // Phys. Rev., 177 (2), pp.567-580 (1969)

21. W.Gerlach, H.Schlangenotto and H.Maeder. On the radiative recombination rate in silicon // Phys. Stat. Sol., A13, p.277-283 (1972)
22. G.Schramm, H.Geitner, and H.A.Schneider. Quantitative analysis of silicon epitaxial layers by photoluminescence // Phys. Stat. Sol., A124, pp.221-233 (1991)

23. D.F.Nelson. Electronic structure in solids. Ed. by E.D.Hademenacis, Plenum Press, 1969, p.122

24. A.P.Gorban, V.P.Kostylyov, A.V.Sachenko. The optimization of $n^{+}-p-p^{+}$-silicon solar cells parameters. Theoretical relations // Optoelectronika i Poluprovodnikovaya Tekhnika, No 34, pp. 63-71 (1999)

25. B.L.Gelmont, V.A. Kharchenko, I.N. Yassievich. An Auger recombination of exciton-impurity complexes // Sov. FTT, 29 (8), pp. 2351-2360 (1987)

26. B.L.Gelmont, N.N. Zinovyev, D.I. Kovalyov, V.A. Kharchenko, I.D. Yaroshetsky, I.N. Yassievich. An Auger recombination of bound excitons induced by ultrasonic phonons // Sov. JETF, 94 (3), pp. 322-335 (1988)

27. V.G. Litovchenko, A.P. Gorban. Physical fundamentals of microelectronic MIS systems. Ed. by Naukova Dumka, Kiev, 1978, $480 \mathrm{p}$.

28. A.Haug. Auger recombination with deep impurities in indirect band gap semiconductors // Phys. Stat. Sol., B108, pp.443-448 (1981) 